\title{
Intestinal Microbiota, Obesity and Insulin Resistance-What Are the Relationships?
}

\author{
Lígia Maria Cordeiro Misurini'1, Nicole do Prado Olbrzymek1, Cicera Aparecida Mendes1, \\ Letícia Godoy Dias Sanderson ${ }^{1}$, Renata Florenzano Flores ${ }^{1}$, Luiz Henrique Corrêa Portari ${ }^{1}$, \\ Luciana Corrêa de Souza Rodrigues ${ }^{1}$, Diana Aristotelis Rocha de Sá1, \\ Larissa Bianca Paiva Cunha de Sá1, Alberto Krayyem Arbex ${ }^{1,2,3}$
}

${ }^{1}$ Endocrinology Division, IPEMED Medical School, São Paulo, Brazil

${ }^{2}$ Diabetology Department, Malteser Krankenhaus St. Franziskus Hospital, Flensburg, Germany

${ }^{3}$ Harvard T. H. Chan School of Public Health, Boston, MA, USA

Email: ligia.misurini@einstein.br,limisurini@hotmail.com

How to cite this paper: Misurini, L.M.C., do Prado Olbrzymek, N., Mendes, C.A., Sanderson, L.G.D., Flores, R.F., Portari, L.H.C., de Souza Rodrigues, L.C., de Sá, D.A.R., de Sá, L.B.P.C. and Arbex, A.K. (2018) Intestinal Microbiota, Obesity and Insulin Resistance-What Are the Relationships? Health, 10, 365-373.

https://doi.org/10.4236/health.2018.104029

Received: March 19, 2018

Accepted: April 6, 2018

Published: April 9, 2018

Copyright $\odot 2018$ by authors and Scientific Research Publishing Inc. This work is licensed under the Creative Commons Attribution International License (CC BY 4.0)

http://creativecommons.org/licenses/by/4.0/

\begin{abstract}
Obesity is a public health problem and its prevalence increases every year, resulting from a complex interaction between genetic, metabolic and environmental factors, as well as cultural influences. The Intestinal Microbiota is composed of trillions of microorganisms by forming a symbiotic relationship with the host and helping the absorption of various nutrients, increasing the extraction of components of the diet, the lipogenesis and intestinal permeability. Studies show that there is difference between the composition of the intestinal microbiota of an obese person and a healthy one. The purpose of this article is to review the literature on the relationship between the Intestinal Microbiota, Obesity and Insulin Resistance and also illustrate some studies on the subject.
\end{abstract}

\section{Keywords}

Obesity, Intestinal Microbiota, Insulin Resistance

\section{Introduction}

The link between Obesity and the composition and function of the Intestinal Microbiota has been studied and cited in recent years [1] [2] [3].

According to data from the Brazilian Ministry of Health, Obesity increased by $60 \%$ in ten years. Between 2006 and 2016, the index rose from $11.8 \%$ to $18.9 \%$. The World Health Organization points to Obesity as one of the greatest public health problems in the world. In 2015, about 2.3 billion adults are overweight; 
and more than 700 million are obese [4].

The human Intestinal Microbiota is made up of approximately 100 trillion bacteria, assisting the human metabolism in general. Some studies show that obesity can be triggered and determined by the proportions of two bacterial phyla of the human intestinal flora-Bacteroidetes (gram negative) and Firmicutes (gram positive) [1]. Some authors still report on the possibility of treating obesity through manipulation of the microbiotic community using probiotics [5] [6] [7].

The Human Intestinal Microbiota consists on about 100 trillion of bacteria that occupy mainly the region of the colon. It suffers diverse dynamical influences in its composition, like age, type of diet, way of delivery, medications in use and current habits of the individuum [7] [8]. Some studies assure that Obesity could be triggered and determined by the proportions presented of 2 Phyla of bacteria of the intestinal flora: Bacteroidetes, gram negative and Firmicutes, gram positive bacteria [8] [9] [10].

Therefore, in view of the great importance of this theme in the current medical situation, this article aims to present a summary of the main studies and discuss the participation of intestinal bacteria in obesity.

\section{Methodology}

In this study, we performed a literature review in the medical literature data available on the web, based on studies from the year 2000 to 2018 that link obesity to the intestinal microbiota. We used forty-one bibliographical references, among which thirteen Brazilian articles and twenty-eight international articles. A thorough review was performed on PubMed and Google Scholar and Scopus databases. The following keywords were used: "probiotics", "gut microbiota", "obesity", "probiotics and obesity".

Seven articles that showed researches in this field were analyzed in this paper. They should have as main goal to characterize the possible change in the intestinal flora associated with corporal weight or with the Body Mass Index (BMI). To evaluate the effects of bacteria, studies should have been performed in humans, in vivo, and not in vitro or in animal models.

\section{Obesity: Definition and Epidemiology}

Chronic metabolic disease characterized by evident clinical manifestations and high rates of prevalence, reaching epidemic proportions. In Brazil, the percentage of obesity rose from $11.8 \%$ in 2006 to $18.9 \%$ in 2016 . The current trend is three out of four Americans will be overweight or obese in 2020, according to the World Health Organization.

Excess weight, be it associated with local or widespread adipose tissue, is related to various morbidities such as metabolic syndrome, type 2 diabetes mellitus, cardiovascular disease and various forms of cancers, thus being a concerning population, generating high cost economical and consequent public health burden. 


\section{Pathophysiology}

Obesity presents a multifactorial etiology, where there is interaction between genetic, metabolic, environmental factors in addition to cultural, psychological and behavioral influences. There may be a better understanding with the neuroendocrine control of appetite, where the key regulator of the energy balance is part of the central melanocortin system, in the hypothalamus. Consisting of two populations of neurons, an appetite stimulating (orexigenic) with Neuropeptide $\mathrm{Y}(\mathrm{NPY})$ and Agouti-related protein (AgRP) and another anorectic composed of Pro-opiomelanocortin (POMC) and the cocaine and amphetamine regulated transcript (CART). The alpha-melanocyte stimulating hormone is one of the products of POMC cleavage and acts as an agonist for MC3-R and MC4-R, the two main melanocortin receptors related to food intake control. The action of Alpha-melanocyte, antagonized by the Agouti protein consists in inhibition of feeding and increasing energy expenditure. Food deprivation increases the expression of the AgRP and NPY genes, and reduces the expression of the POMC and CART genes. Therefore, the balance of hypothalamic peptides, orexigenic and anorectics, are crucial for an adequate control of food intake [3].

Incisive and determinants in obesity, ghrelin and leptin are gaining day-to-day research to deepen the understanding in their functions. The ghrelin, secreted mainly by the cells of the stomach, acts on the central nervous system, signaling the need to ingest food.

Its role in the control of metabolism has recently been studied, and it has been observed that ghrelin administration in rats induces the behavior of food intake, reduction in metabolic spending and obesity. The secretion of ghrelin is inhibited by the ingestion of nutrients, as these stimulate the secretion of several other intestinal and pancreatic hormones that would control their release.

The ghrelin stimulates the release of hypothalamic peptides (NPY and AgRP the) which are potent stimulators of appetite, i.e. Orexigenic [3].

Leptin's name is derived from the Greek word leptos, which means "thin". Leptin is a hormone comprised of 167 with amino acids, transcribed from the ob gene, which was originally cloned in mice. The mutation of this gene, or its dysfunction, causes severe obesity and type II diabetes in these animals. The human leptin gene is located on chromosome $7 \mathrm{q} 31$ and its DNA has more than 15,000 pairs of bases and there are three exons. Leptin is produced mainly in white adipose tissue and, when injected into ob/ob mouse (that has genetic deficiency of this peptide) reduces the consumption of food and increases energy expenditure. On the other hand, when leptin is injected in the $\mathrm{db} / \mathrm{db}$ mouse (featuring leptin receptor deficiency) there is no weight loss or decreased energy consumption [4].

\section{Insulin Resistance}

Situation in which insulin does not have its full activity, where there is evidence 
that a chronic inflammatory process could represent the triggering factor at the origin of this and eventually the onset of type 2 diabetes. There are two main adipocytes in the human body, the subcutaneous adipocytes and the visceral adipocytes. The first is smaller, multiplies easily, stores large capacities of free fatty acids and produces few inflammatory cytokines [5].

In contrast, the visceral adipocytes are large cells that rarely divide, are metabolically more active, accumulate less free fatty acids and have great ability to secrete inflammatory cytokines. The excess of pro-inflammatory cytokines (especially TNF-alpha and IL-6) result in inflammation in adipose tissue that spreads to a global systemic inflammation associated with the development of obesity and insulin resistance. Therefore, overfeeding, especially rich in saturated fats, and also environmental influences, such as chronic stress, could, together, result in increased secretion of these cytokines and consequently development of insulin resistance and obesity [5] [6].

\section{Intestinal Microbiota}

The intestinal microbiota is defined as a set of micro-organisms, including bacteria, Archaea, fungi, and viruses that inhabit the human gut in a dynamic and stable manner, establishing a symbiotic relationship.

Spezia et al., 2009, say that these microorganisms proliferate mostly in the region of colon, due to the nutritional supply available, lack of intestinal secretion and slow peristaltic waves [7].

Penteado J.O., 2017 et al. claim that the human intestinal microbiota, stores up to 100 trillion bacteria. There are 2 predominant phyla in adults, highlighting the Bacteroidetes, composed of gram-negative and Firmicutes, gram-positive. The Firmicutes are divided into 3 classes: Bacilli (voluntary or compulsory aerobic, mainly involving Lactobacillus and Enterococcus); Mollicutes and Clostridia (anaerobes) [8]. Secondly there are the species of Bifidobacterias (gram-positive), Eubacteria, Fusobacterias.

It was believed that at birth the child gastrointestinal tract was sterile. Boulangé C.I., 2016 et al. say that gut colonization begins in prenatal life, through the transmission of bacteria from the mother to the fetus through the placenta. The route of delivery is an important factor in this colonization, since through the vaginal birth, the child comes into contact with the vaginal and perineal microbiota of the mother [9].

In cesarean section, this does not occur and one acquires bacteria from the contact with the environment, and especially with the type of diet offered. Highlighting the supply of breast milk, which favors the appearance of Bifidobacterias [9].

By the age of 2 to 5 years of age, the child presents a micro-pattern very similar to the adult, with small fluctuations [10].

Thus, such factors may lead to overgrowth of bacteria, so that transient microbiota passes the overlap to the resident, changing gastrointestinal function. 
The elderly have a higher number of anaerobic bacteria, increasing the number of pathogenic bacteria (enterobacteria and clostridia), decreasing the number of protective bacteria such as Bifidobacteria.

The intestinal microbiota has great power to adapt the diet held, producing different metabolic responses.

Predominant consumption of saturated fats and polyunsaturated fats set up the conditions that lead to the predominance of Firmicutes bacteria in the intestine. That then leads to a situation where greater absorption and storage of nutrients, favoring obesity. Notes that this type of diet alters intestinal permeability increases the presence of bacterial lipopolysaccharides (LPS) in the lumen and predisposes to subclinical chronic inflammation. In the liver, they act by inducing local inflammation, decrease insulin sensitivity and favor the infiltration of macrophages. In adipose tissue and muscle there is also the increase of lipoprotein lipase [11].

On the other hand, high-fiber diet (fruit, vegetables) are associated with proliferation of Bacteroidetes and a lesser proportion, Clostridium and Bifidobacterium. They improve metabolism, the immune system, endocrine signaling, brain function. These effects are associated to the increase of short chain fatty acids, fruits of food fermentation, decreasing the local $\mathrm{pH}$, favoring the relative proliferation of Bacterioides [12].

Another observation is the presence of toll-like receptors (TRL4), which are membrane receptors present in intestinal cells, which when activated recognize fatty acids and lipopolysaccharides that induce insulin resistance and obesity. Andrade V.L. et al. showed that rats submitted to a high fat diet had a higher expression of TRL4, suggesting an association of the activation of this receptor with increased hunger and accumulation of body fat. There is also the activation of toll-like receptors (TRL5) which is mainly related to changes in insulin sensitivity.

\section{Linking Obesity and Insulin Resistance to Intestinal Microbiota}

The intestinal microbiota emerged as a new contribution in research on obesity and its comorbidities such as insulin resistance, type 2 diabetes mellitus and others. The pathophysiological processes remain partially obscure in changing the composition and function of intestinal microbiota.

Some mechanisms have been proposed in an attempt to explain the relationship between obesity and intestinal flora such as: the function of the intestinal flora in the energy extraction of nondigestible polysaccharides, the modulation of lipopolysaccharide levels by the intestinal flora, which triggers chronic subclinical inflammation that leads to obesity and diabetes and there is also the mechanism that proposes that the intestinal flora can induce the gene regulation of the host systems that modulate how energy is spent and stored.

In a study by Backhed et al. in 2004, where they analyzed for 14 days skinny 
mice colonized by microbiota of genetically obese mice, obtained data that showed modest fat gain in those mice, as well as greater extraction of energy from food. When colonized with microbiota from genetically obese mice, they showed a $60 \%$ increase in body fat. Moreover, insulin resistance increased despite reducing food intake, producing a considerable increase in body fat, despite the reduction in food consumption [13].

When the diet offered was high in fat, rats showed increased endotoxin, proinflammatory cytokines, increased macrophage infiltration, inflammation of the intestinal microbiota, with consequent imbalance of the intestinal flora, induced growth of enterobacteria and increase in relation to the firmicutes and bacterioides [13].

It was also observed that non-germ rats were protected from obesity, insulin resistance, dyslipidemia and non-alcoholic hepatic steatosis (NASH), even when fed with high-fat diet [13].

Bäckhed et al. also showed that germ-free mice need to consume approximately $30 \%$ more calories to maintain body weight similar to those colonized with the intestinal microbiota [13].

In another study, where the animal model was also used, Ley et al. (2005) compared the microbiota of the intestine of lean mice with obese mice. This showed that the relative amount of Bacteroidetes in ob/ob (obese) mice was 50\% lower, whereas that of Firmicutes was 50\% higher [14].

It is believed that the intestinal microbiota contributes to metabolic diseases by stimulating low-grade inflammation leading to obesity. The expression of genes involved in energetic homeostasis, lipid metabolism and mitochondrial metabolism in different parts of the intestine, as well as in liver and adipose tissues, is markedly different in rats without germ as well as the conventional one [15].

The communication of the microbiota with the immune system is due to the increase of the innate immune response and the control of the inflammation through the pathways regulated by toll-like receptors (TRL). Activation of TRL5 in epithelial or myeloid cells affects the intestinal microbiota structure, which regulates appetite, weight gain and insulin sensitivity [15].

Recent studies suggest that Bifidobacteria and Bacteroides ssp appear to be able to protect against weight gain, giving rise to the "microbial hypothesis" of obesity, which may determine important therapeutic implications in the future.

There are multiple metabolic mechanisms that associate microbiota, obesity and hepatic steatosis. We can relate the bacterial fermentation of polysaccharides that were not previously digested by man, which leads to the production of monosaccharides and short-chain fatty acids (SCFAs). These are substrates of colonocytes and precursors of cholesterol and fatty acids, favoring participation in hepatic gluconeogenesis; SCFAs bind to specific receptors in intestinal endocrine cells, known as GPR41 and GPR43, which are G protein conjugates.

Continuing the metabolic mechanisms involved, the microbiota has the 
Table 1. Research showing the relationship between obesity and the intestinal microflora.

\begin{tabular}{ccc}
\hline Study & Design & Objective \\
\hline
\end{tabular}

1) Bäckhed et al. (2004) [13]

2) Turnbaugh et al. (2006) [17]

3) Collado et al. (2008) [21]

4) Ley et al. (2005) [14]

5) Kalliomäki et al. (2008) [18]

6) Santa Cruz et al. (2010) [19]

7) Jumpertz et al. [20]
Rats with sterile microbiota and conventional mice. Time period: 2 weeks

Obese rats (ob:ob) and lean mice

18 pregnant women with overweight and 36 with normal weight. Follow up for 9 months.

Genetically obese mice (ob/ob) and lean mice monitoring for 2 weeks

25 obese and overweight children 24 with normal weight Ages between 4 and 7 years

Intestinal Microbiotic Analysis of pregnant women (50) obese (16) and non-obese (34)
Evaluation of the role of the microbiota in the regulation of nutrient absorption in twelve lean and nine obese individuals subject to a daily diet of different energy value
Evaluate the colonization and change of intestinal microflora of mice

Analyze the relationship between obesity and intestinal microbiota

To evaluate the composition of the intestinal microbiota

To compare the intestinal microbiota of ob/ob versus lean mice

To establish whether the composition of the early intestinal microbiota may influence body weight in childhood

To analyze the composition of the intestinal microbiota of healthy and obese pregnant women, to establish the relationship between the microbiota and weight gain during pregnancy

Evaluation of the regulation of nutrient bioavailability
Outcomes and Comments

It showed that the microbiota transported from obese rats to the sterile mice not only increased the absorption of calories from the diet, but also increased the adiposity in these rats, even maintaining the standard diet.

Microbiota of obese $\geq$ ability to store energy

Microbiota of overweight women $\geq$ concentration of Bacterioides and Staphylococcus The greater the overweight $>$ the concentration of Bacteroidetes, Clostridium and Staphylococcus

Obesity rats colonized with microbiota had modest fat gain and extracted more energy from their diet.

Microbiota of obese and overweight $\geq$ concentration of

Staphylococcus aureus Microbiota of children with normal weight $\geq$ number of Bifidobacteria

Microbiota of obese pregnant women is different from the microbiota of previously healthy pregnant women

The change in nutrient load-induced microbiota is directly related to feces in lean individuals and that a $20 \%$ increase in Firmicutes and a corresponding decrease in Bacteroidetes was associated with an increased energy absorption of $150 \mathrm{kcal}$

function of regulating some host genes responsible for lipid deposition in adipocytes; In addition to increasing the hepatic capture of monosaccharides from the portal circulation potentiating lipogenesis [16].

The main studies showing a possible relationship between Obesity and the Intestinal Microflora are shown on Table 1. 


\section{Conclusions}

Studies suggest that there are relevant differences between the composition of the intestinal microbiota of an obese person and that of a healthy one.

These analysed articles suggest that the intestinal microbiota may participate in the pathophysiology of obesity. Despite the great interest in the subject, these studies are so far small and some questions still remain unanswered: Could there be a treatment for obesity by manipulating the intestinal microbiota? Could a change of the intestinal flora of a patient lead to weight loss without dieting? How could we avoid or stimulate the change of intestinal flora in our environment? Why and how do lean patients preserve their intestinal bacteria over the years despite habit changes?

Therefore, the role of the gut microbiota in obesity remains a current hot topic. This review suggests a need for further research of this theme by clinical trials, regarding a possible new trait in understanding and treating obesity through the manipulation of the gut microbiota.

\section{References}

[1] Pistelli, G.C. and Costa, C.E.M. (2010) Bactérias Intestinais e Obesidade. Bacterias Intestinais e Obesidade. Revista Saúde e Pesquisa, 3, 115-119.

[2] Martinez, R.C.R. (2015) Scientific Evidence for Health Effects Attributed to the Consumption of Probiotics and Prebiotics: An Update for Current Perspectives and Future Challenges. British Journal of Nutrition, 114, 1993-2015. https://doi.org/10.1017/S0007114515003864

[3] Coll, A.P., Farooqi, I.S. and O'rahilly, S. (2007) The Hormonal Control of Food Intake. Cell, 129, 251-262. https://doi.org/10.1016/j.cell.2007.04.001

[4] WHO (2018) Obesity and Overweight, Fact Sheet. http://www.who.int/mediacentre/factsheets/fs311/en/

[5] Pliquett, R.U., et al. (2006) The Effects of Insulin on the Central Nervous System-Focus on Appetite Regulation. Hormone and Metabolic Research, 38, 442-446. https://doi.org/10.1055/s-2006-947840

[6] Saad, M.J., Santos, A. and Prada, P.O. (2016) Vinculando Microbiota Gut e Inflamação à Obesidade e à Resistência à Insulina. Fisiologia (Bethesda), 31, 283-293.

[7] Spezia, G., Silva, L.T., Santos, S.P., Liberati, R. and Navarro, F. (2009) Microbiota intestinal e sua relação com a obesidade. Revista Brasileira de Obesidade, Nutrição e Emagrecimento, 3, 260-267.

[8] Penteado, J.O., Salgado, R.G.F. and Barlen, E. (2017) A eficácia do tratamento da Obesidade através de transplante da microbiota fecal de indivíduos magros. Vitalle, 29, 46-53.

[9] Boulangé, C.L., Neves, A.L., Chilloux, J., Nicholson, J.K. and Dumas, M.E. (2016) Impact of the Gut Microbiota on Inflammation, Obesity, and Metabolic Disease. Genome Medicine, 8, 42. https://doi.org/10.1186/s13073-016-0303-2

[10] Collado, M.C., Salminen, S. and Isolauri, E. (2008) Early Differences in Fecal Microbiota Composition in Children May Predict Overweight. American Journal of Clinical Nutrition, 87, 534-538. https://doi.org/10.1093/ajcn/87.3.534

[11] Trabulsi, L.R. and Sampaio, M.C. (2008) Microbiota Normal do Corpo Humano. In: Microbiologia, 5a edição, Atheneu, Rio de Janeiro, 103-110. 
[12] Kim, K.A., Gu, W., Lee, I.A., Joh, E.H. and Kim, D.H. (2012) High Fat Diet-Induced Gut Microbiota Exacerbates Inflammation and Obesity in Mice via the TLR4 Signaling Pathway. PLoS One, 7, e47713. https://doi.org/10.1371/journal.pone.0047713

[13] Backhed, F., Ding, H., Wang, T., Hooper, L.V., Koh, G.Y., Nagy, A., et al. (2004) The Gut Microbiota as an Environmental Factor That Regulates Fat Storage. Proceedings of the National Academy of Sciences of the United States of America, 101, 15718-15723. https://doi.org/10.1073/pnas.0407076101

[14] Ley, R.E., Turnbaugh, P.J., Klein, S. and Gordon, J.I. (2006) Microbial Ecology: Human Gut Microbes Associated with Obesity. Nature, 444, 1022-1023. https://doi.org/10.1038/4441022a

[15] Larsson, E., Tremaroli, V., Lee, Y.S., Koren, O., Nookaew, I., Fricker, A., et al. (2012) Analysis of Gut Microbial Regulation of Host Gene Expression along the Length of the Gut and Regulation of Gut Microbial Ecology through MyD88. Gut, 61, 1124-1131. https://doi.org/10.1136/gutjnl-2011-301104

[16] Gibson, G.R., Probert, H.M., Loo, J.V., Rastall, R.A. and Roberfroid, M.B. (2004) Dietary Modulation of the Human Colonic Microbiota: Updating the Concept of Prebiotics. Nutrition Research Reviews, 17, 259-275. https://doi.org/10.1079/NRR200479

[17] Turnbaugh, P.J., et al. (2006) An Obesity-Associated Gut Microbiome with Increased Capacity for Energy Harvest. Nature, 444, 1027-1031.

[18] Kalliomaki, M., Collado, M.C., Salminem, S. and Isolauri, E. (2008) Early Differences in Fecal Microbiota Composition in Children May Predict Overweight. The American Journal of Clinical Nutrition, 87, 534-538.

[19] Santacruz, A., Collado, M.C., Garcia-Valdes, L., Segura, M.T., Martin-Lagos, J.A., Anjos, T., et al. (2010) Gut Microbiota Composition Is Associated with Body Weight, Weight Gain and Biochemical Parameters in Pregnant Women. British Journal of Nutrition, 104, 83-92. https://doi.org/10.1017/S0007114510000176

[20] Jumpertz, R., Le, D.S., Turnbaugh, P.J., Trinidad, C., Bogardus, C., Gordon, J.I., et al. (2011) Energy-Balance Studies Reveal Associations between Gut Microbes, Caloric Load, and Nutrient Absorption in Humans. The American Journal of Clinical Nutrition, 94, 58-65. https://doi.org/10.3945/ajcn.110.010132

[21] Collado, M.C., Isolauri, E., Laitinen, K. and Salminen, S. (2008) Distinct Composition of Gut Microbiota during Pregnancy in Overweight and Normal-Weight Women. The American Journal of Clinical Nutrition, 88, 894-899. https://doi.org/10.1093/ajcn/88.4.894 